\title{
Complex antibodies in the Guillain Barré syndromes provide a simple explanation for the plurality of clinical presentations
}

\author{
Dr Simon Rinaldi \\ Academic Clinical Lecturer, Nuffield Department of Clinical Neurosciences \\ Neuroscience Tutor, Exeter College \\ University of Oxford \\ Level 6, West Wing, John Radcliffe Hospital \\ Oxford OX3 9DU \\ Email: simon.rinaldi@ndcn.ox.ac.uk
}

A strong argument can be made for using the plural of the epnoymous term when referring to the acute post infectious polyradiculoneuropathies. The Guillain Barré syndromes thus (GBS) include axonal, demyelinating and paranodonodopathy forms, defined electrophysilogically or pathologically. Clinically recognised variants can be limited to specific body regions (such as facial diplegia or paraplegic GBS) and prefernetially or exclusively affect specific nerve types (pure motor, sensory, acute pandysautonomia). Antibodies directed against the ganglioside GQ1b are strongly associated with the GBS variant known as Miller Fisher syndrome (MFS), yet are also found in formes fruste of MFS, Bickerstaff's brainstem encephalitis, and the regional pharyngeal-cervical-brachial variant. An explanation for the plurality of clinical presentations associated with this single antibody has thus far proved elusive.

The idea that distinct epitopes might be formed by "clustered saccharide patches" composed of different oligosaccharide chains is not a new one (Varki, 1994). Indeed the related observation that the presence of a second ganglioside in the cell membrane can prevent binding to the first was intially made over 20 years ago in melanoma cell lines (Lloyd et al., 1992). Following the detection of ganglioside complex (GSC) antibodies in the serum of patients with GBS by Kusunoki and colleagues (Kaida et al., 2004), interest in this area has grown. Such 'complex enhanced' antibodies where demoonstrated to recognise overlapping portions of 2 different gangliosides, binding weakly or not at all in the presence of only one. Ganglioside complexes were subsequently shown to have a significant influence on the binding affinity of other lectins such as bacterial toxins and the sialic acid binding immunorecptor famility known as SIGLECs (Rinaldi et al., 2009). Furthermore, anti-GM1 antibodies which additionally interact with GM1-complexes ('complex independent') are able to cause nerve injury in a model system, whereas those with GM1 reactivity alone ('complex attenuated') are pathologically inert (Greenshields et al., 2009). When appleied to clinical cohorts, immnoassays using complexes can provide increased sensitivity and/or have revealed associations between specific anti-complex antibodies and certain clinical features (Kaida et al., 2007; GalbanHorcajo et al., 2012; Rinaldi et al., 2013).

The current study from Professor Yuki's prolific group has collected a very impressive 915 serum samples, from over 10,000 tested, with anti-GQ1b or anti-GT1a ganglioside reactivity. Attesting to the specificty of these anitbodies, all positive samples were drawn from patients with an ultimate 
diagnosis of MFS or one of the related anti-GQ1b syndromes. The authors sought to determine whether the fine specificty of these antibodies, with respect to their ganglsiode complex binding capabilities, was associated with specific clincal features of the anti-GQ1b syndromes. Applying a statistical definition to both the definition of complex enhanced or attenuated binding, as well as to the serological-clinical associations, a large number of different neurological signs have been shown to be associated with specific anti-complex antibodies. Whilst the number of new associations demononstrated is initially somewhat bewildering, and the serological segregation between patients with and without specific featurs incomplete, the underlying concept is as clear as it is thought provoking.

As the authors point out, the implication is that neural membranes at different regions within the nervous system have distinct, ganglioside-complex antigenic profiles. It has so far been unclear why anti-GM1 antibodies are associated with pure motor syndromes, despite sensory nerves containing abundant GM1 antigen. The current study suggests that this might also relate to a difference in GM1 complexes between the nerve types. Localised differences and inter-subject variations in ganglioside-complex expression may also be of relevance to the breakdown in self-tolerance required to induce GBS. If specific complexes are only generated in immunologically privileged parts of the nervous system they may not be recognised as self and thus predisposed to autoimmune attack. The challenges now are to demonstrate the variability of ganglioside-complex expression in different tissues, and to understand the factors governing their differential formation. Whilst immunohistochemical studies have been proposed, the power of cutting-edge membrane imaging techniques may be required to effectively address this first question.

\section{References}

Galban-Horcajo, F., Fitzpatrick, A.M., Hutton, A.J., Dunn, S.M., Kalna, G., Brennan, K.M., Rinaldi, S., Yu, R.K., Goodyear, C.S., and Willison, H.J. (2012). Antibodies to heteromeric glycolipid complexes in multifocal motor neuropathy. Eur. J. Neurol. Off. J. Eur. Fed. Neurol. Soc.

Greenshields, K.N., Halstead, S.K., Zitman, F.M.P., Rinaldi, S., Brennan, K.M., O'Leary, C., Chamberlain, L.H., Easton, A., Roxburgh, J., Pediani, J., et al. (2009). The neuropathic potential of anti-GM1 autoantibodies is regulated by the local glycolipid environment in mice. J. Clin. Invest. 119, 595-610.

Kaida, K., Morita, D., Kanzaki, M., Kamakura, K., Motoyoshi, K., Hirakawa, M., and Kusunoki, S. (2004). Ganglioside complexes as new target antigens in Guillain-Barre syndrome. Ann.Neurol. 56, 567-571.

Kaida, K., Morita, D., Kanzaki, M., Kamakura, K., Motoyoshi, K., Hirakawa, M., and Kusunoki, S. (2007). Anti-ganglioside complex antibodies associated with severe disability in GBS.

J.Neuroimmunol. 182, 212-218.

Lloyd, K.O., Gordon, C.M., Thampoe, I.J., and DiBenedetto, C. (1992). Cell surface accessibility of individual gangliosides in malignant melanoma cells to antibodies is influenced by the total ganglioside composition of the cells. Cancer Res 52, 4948-4953.

Rinaldi, S., Brennan, K.M., Goodyear, C.S., O'Leary, C., Schiavo, G., Crocker, P.R., and Willison, H.J. (2009). Analysis of lectin binding to glycolipid complexes using combinatorial glycoarrays. Glycobiology 19, 789-796. 
Rinaldi, S., Brennan, K.M., Kalna, G., Walgaard, C., van Doorn, P., Jacobs, B.C., Yu, R.K., Mansson, J.E., Goodyear, C.S., and Willison, H.J. (2013). Antibodies to Heteromeric Glycolipid Complexes in Guillain-Barré Syndrome. PLoS ONE 8, e82337.

Varki, A. (1994). Selectin ligands. Proc.Natl.Acad.Sci.U.S.A 91, 7390-7397. 